\title{
Dossier Familias Indígenas. En memoria de Rolando Mellafe y Enrique Tandeter
}

\section{Dossier Indigenous Families. In memory of Rolando Mellafe and Enrique Tandeter}

\author{
Xochitl Inostroza ${ }^{1}$ y Juan Pablo Ferreiro ${ }^{2}$
}

El dossier Familias Indigenas contiene algunas de las exposiciones que integraron el simposio "Historia de familias indígenas en perspectiva interdisciplinaria: materiales, documentos, métodos", realizado en la Universidad de Santiago de Chile durante al año 2015, en el marco del IV Congreso Internacional de Ciencias, Tecnologías y Culturas. Por este motivo, agradecemos en primer lugar a los profesores Eduardo Devés y Fernando Estenssoro, coordinadores generales del congreso, por darnos el espacio para este encuentro que nos permitió realizar dos jornadas de diálogo y reflexión interdisciplinaria sobre la familia indígena, bajo condiciones ideales para una reunión académica, contando con tiempo suficiente para cada exposición, generando un diálogo fructífero a partir de las diferentes investigaciones. Agradecemos también a los editores de la revista Estudios Atacameños. Arqueología y Antropología Surandinas, Gonzalo Pimentel y Helena Horta, quienes nos dieron el espacio para publicar algunas de estas intervenciones. Agradecemos también al proyecto FONDECYT 1130667 y a su investigador responsable, Jorge Hidalgo, por apoyar la organización de estos simposios.

Este encuentro en Santiago fue la continuación de un diálogo que se inició en Arica durante el año 2014, el cual dio origen a un primer dossier publicado en Surandino Monográfico (UBA, 2017). Para nosotros, organizadores de este encuentro, era necesario generar espacios que nos permitieran compartir las actuales investigaciones sobre la historia de la familia indígena que se ha ido abordando en los últimos años desde diversas disciplinas. Esperamos seguir generando nuevas instancias para continuar con este diálogo, integrando otras investigaciones de diferentes áreas de Latinoamérica.

El diálogo en torno a las familias indígenas surge del reconocimiento empírico y analítico de una gran, y aún no completamente comprendida, diversidad de formas y lógicas culturales que impiden continuar postulando la existencia de modelos únicos (mediterráneo, familia nuclear, etc.), aunque sí reconociendo y explorando la extensión de modelos hegemónicos en determinadas épocas y regiones. Es por ello que la convocatoria buscó integrar diversas disciplinas para reflexionar respecto de la familia indígena en perspectiva histórica, con énfasis en los métodos, materiales

1 Proyecto FONDECYT Postdoctoral 3180287. Centro de Estudios Culturales Latinoamericanos, Universidad de Chile. Av. Ignacio Carrera Pinto 1025, Santiago, CHILE. Email: xochitl@uchile.cl

2 CONICET/Universidad Nacional de Jujuy. El Naranjero 384, 4600 San Salvador de Jujuy, ARGENTINA. Email: tresaguilhones@gmail.com 
y fuentes documentales con los que trabajamos, mediante los cuales se buscaba establecer marcos comparativos respecto de las estrategias metodológicas aplicadas a distintos grupos del continente.

Los trabajos que integran este dossier se plantean entonces desde una mirada interdisciplinaria, y a partir de distintos escenarios. Es sintomático que varios de ellos utilicen registros parroquiales como fuentes de información para construir sus relatos sobre familias indígenas. Pues bien, en Chile, desde las investigaciones de Rolando Mellafe estos documentos se han constituido como uno de los espacios privilegiados de la historia social, permitiendo observar a las familias del pasado desde el punto de vista sociodemográfico. En esta ocasión, estos materiales se integran al análisis de registros históricos que se enmarcan entre los siglos XVII y XX. En algunos de ellos también se dejan ver las huellas que dejó el trabajo pionero de Enrique Tandeter, quien utilizando este mismo tipo de registros reconstruyó genealogías de familias indígenas de Sacaca y Acasio, impulso al que en esta ocasión se han sumado viejas y nuevas técnicas provenientes de la demografía histórica y el análisis de redes sociales, principalmente.

Tres trabajos se podrían definir como metodológicamente más tradicionales. El de Ignacio Chuecas, que utiliza documentación proveniente de archivos de la administración colonial para dar cuenta de las transformaciones que sufren las familias de una reducción ubicada en la frontera de la Araucanía. El artículo de Simón Urbina, Leonor Adán, Constanza Pellegrino y Roberto Izaurieta analiza arqueológicamente una treintena de sitios en donde los patrones formales de las estructuras domésticas resultan indicadores de cambios en la organización/estructura social de las comunidades tarapaqueñas. El trabajo de Daniela Salvucci acude a las tradicionales técnicas etnográficas para dar cuenta de las dinámicas de las actuales familias de Jasimaná, en el Noroeste Argentino (NOA). Los tres trabajos llegan a conclusiones novedosas que escapan de las formas tradicionales con las que se ha abordado la familia desde la historia, la arqueología y la antropología. Por otra parte, los artículos de Jaime Valenzuela (siglo XVII), Xochitl Inostroza (siglos XVIII-XIX), Juan Pablo Ferreiro (siglo XIX) y Federico Fernández (siglo XX), pese a que están sustentados en el análisis de libros parroquiales, utilizan metodologías muy diversas, lo que en sí mismo constituye un diálogo sobre la gran variedad de posibilidades que existen para abordar un mismo cuerpo documental.

Todos los artículos de este dossier representan un aporte original a la historia de las familias indígenas; tanto en su contenido como en evidenciar el dinamismo de la familia, relevando la importancia de su análisis histórico, pero también en sus respectivas propuestas teóricas y/o metodológicas que sin duda dan cuenta de la importancia de la interdisciplinariedad, necesarias para abordar estas temáticas. Por ello, el orden de los artículos responde a una organización espacial por una parte, y cronológica por otra, y no a una clasificación disciplinaria.

El dossier se inicia con los trabajos de Jaime Valenzuela y de Ignacio Chuecas, referidos ambos a familias de origen mapuche. El artículo de Valenzuela se titula "El matrimonio como espacio de 'desfragmentación' entre mapuche-huilliches desna- 
turalizados a Santiago de Chile durante la segunda mitad del siglo XVII (16691678)". El autor muestra cómo a partir de las razias esclavistas de la guerra fronteriza y traslados forzosos a la ciudad de Santiago, mapuches y huilliches meridionales experimentaron procesos de desarraigo y destrucción de los lazos familiares. Analiza los registros de matrimonios de sujetos de origen mapuche que se casaron en la parroquia El Sagrario durante la década de 1669-1678, para dar cuenta acerca de cómo sus experiencias de desarraigo "se transforman en signos de su identidad y memoria”, lo cual se traduce en la conformación de nuevas familias a partir de un proceso de recomposición o "desfragmentación". El trabajo de Ignacio Chuecas, titulado "Santa Fe mo (Inche ñi mapu mo cay). Vida familiar en la reducción fronteriza de Santa Fe (siglo XVIII)", tiene su escenario en las reducciones ubicadas a orillas del río Biobío, que para el autor representan un espacio negociado donde las familias indígenas desplegaron diversas estrategias para enfrentar al sistema colonial hispano. Una de las particularidades del proceso dice relación con la inestabilidad en el asentamiento, lo que, sin embargo, no se traduce en el abandono de tradiciones y modos de vida, sino más bien da lugar a prácticas familiares híbridas, como la de pagas a la usanza por el precio de la novia, el matrimonio canónico cristiano, las funciones familiares de los caciques gobernadores, en un modelo de propiedad familiar donde confluyen modalidades indígenas y europeas.

Continúan el trabajo de Urbina, Adán, Pellegrino e Izaurieta, así como el artículo de Inostroza, que están circunscritos a espacios del norte chileno, específicamente a las regiones de Tarapacá y de Arica y Parinacota, respectivamente. El primero, titulado "Historia arquitectónica de Tarapacá: estrategias residenciales y formación de asentamientos, siglos X AC a XVII DC (Andes Centro Sur)", sintetiza el estudio de patrones arquitectónicos residenciales para el área geográfica de Tarapacá entre los siglos X AC y XVII DC, intentando mostrar cómo la historia de la construcción habitacional es la historia organizacional de sus habitantes. El cambio de una estrategia residencial neolocalista, que permite la formación de asentamientos dispersos, a una de aglomeración posmarital que asegura la aglomeración de las unidades familiares descendientes, parece ser el punto crucial en la formación de los primeros núcleos aldeanos durante el período Formativo Temprano y que, con posterioridad al abandono de estos últimos, alcanzaría límites críticos en los extensos barrios de "Caserones", revelando altos grados de competencia y disputas políticas entre familias extensas y linajes hereditarios dentro de la región. El de Inostroza, es una propuesta metodológica titulada "Complementariedad de métodos y fuentes en el estudio histórico de familias indígenas. Belén, Altos de Arica (1750-1820)”, que surge del método demográfico francés conocido como "reconstitución de familias", pero que integra otros métodos y documentos que le permiten una comprensión global de los variados aspectos que intervienen en la conformación de las familias del pueblo de Belén, en los Altos de Arica. A partir de ellos, distingue fenómenos tales como la presencia de familias compuestas, dos posibles casos de poliginia, la tendencia masculina a la endogamia al interior del pueblo, las posibles preferencias que regulan los matrimonios, destacando las diferencias que se observan entre primeras y segundas nupcias; además observa las dificultades demográficas y sociales que le llevan a hipotetizar respecto del rol que jugaron estos factores en contra de la familia extensa y a favor del parentesco ritual. 
Finalizan el dossier los trabajos de Juan Pablo Ferreiro, Federico Fernández y Daniela Salvucci, enmarcados espacialmente en el NOA y que temporalmente son los más actuales, por lo que presentan algunos puntos en común. El artículo de Ferreiro busca reconocer y analizar las configuraciones que desarrollan algunos vínculos de parentesco, y diversos pisos ecoambientales, que van desde las selvas húmedas de las yungas en el meridional valle de La Candelaria $(1000 \mathrm{msnm})$ hasta los valles altos de la zona de Santa Ana (4700 msnm), en el extremo septentrional del actual departamento de Valle Grande, provincia de Jujuy. Está centrado en el análisis de lo que llama el "frente de parentesco Apaza", a partir de métodos provenientes del análisis de redes de parentesco y la Teoría de Grafos. En él, el matrimonio entre sujetos campesinos muestra algunas similitudes con las de otras familias jujeñas, sobre todo en cuanto a la "endogamia patrimonial" como estrategia de supervivencia y mantención de privilegios, sustentada en una memoria al parecer, selectiva. Sin embargo, en una población estabilizada demográficamente durante varios siglos, con casi nulo aporte migratorio y un alto grado de aislamiento físico provocado por lo abrupto de su territorio, no poder recordar más de tres generaciones ascendentes no parece solo una cuestión de mero olvido, sino que respondería más bien a una estrategia de sobrevivencia. El artículo de Fernández, titulado "Redes de capital social familiar en la región oriental de Jujuy (Argentina) durante las tres primeras décadas del siglo XX", analiza la utilización de la categoría capital social, y sus vinculaciones posibles con los procesos de acumulación relacional y movilidad espacial entre familias extensas con residencias múltiples dentro de la región de Valle Grande, provincia de Jujuy. Los resultados obtenidos tras el ordenamiento métrico y gráfico de los datos dan cuenta de una serie de pautas vinculares factibles de ser descritas bajo la categoría de capital social familiar. El autor identifica como los principales hilos socio-parentales locales la marcada endogamia de pueblo y la familia extensa con residencia múltiple, donde las relaciones de apadrinamientos bautismales se comprenden a la manera de una red relacional duradera en el tiempo y de carácter afectivo (capital social familiar), basada en la movilización y mantenimiento de recursos y/o servicios (capital simbólico y económico), los que exigieron una inversión temporal y económica por parte de quienes conformaban y sustentaban esta red, contexto en el cual las relaciones fuertes entre apadrinamientos extralocales e hijos naturales pueden ser interpretadas como una estrategia vincular llevada a cabo en muchos casos (no en todos) por mujeres sin pareja progenitora. El escrito de Salvucci, titulado "Prácticas y categorías de parentesco en Jasimaná, noroeste andino de Argentina”, busca analizar, desde una perspectiva de antropología cultural, las categorías émicas y las prácticas del parentesco, afirmando que las categorías antropológicas no siempre logran explicar las situaciones concretas y que los conceptos de "grupo doméstico" y "red familiar" solo tienen sentido en una perspectiva de traducción, como punto de partida para acercarnos a comprender las relaciones locales y las categorías émicas; de esta manera, refuerza la perspectiva culturalista, proponiendo que tanto en Jasimaná como en otras comunidades de la macro área andina, el ámbito del parentesco se fundamenta en procesos y acciones como las conectadas a la "cocina", a la "casa", al "criar" y al "reconocerse". 